\title{
Tertiary Protein Structure
}

National Cancer Institute

\section{Source}

National Cancer Institute. Tertiary Protein Structure. NCI Thesaurus. Code C14064.

Tertiary structure describes the folding of the polypeptide chain to assemble the

different secondary structure elements in a particular arrangement. 